\title{
Antiurolithiatic Activity of Pisonia Alba Leaves in Experimental Animal Model (Albino Rats)
}

\author{
Kavitha Rani Mari, Suriyavathana Mthukrishnan, Punithavathi Manogaran, Anandhi Eswaran
}

\begin{abstract}
Pisoniaalbais a medicinal plant used widely has a broad variety of pharmacological uses. The current research explored the acute toxicity of an ethanol sample of Pisoniaalba (Family: Nyctaginaceae) Ethanol Leaves Extract Pisoniaalba(ELPA) and Pisoniaalba(ALPA) Aqueous Leaves Extract at a dose of 1000mg / $\mathrm{kg}$ and comparison of urinary amount and electrolyte amount as parameters of evaluation. On the other side, urolithiasis caused by ethylene glycol $(0.75 \% v / v$ in drinking water for 28 days) was used for the same ELPA and ALPA with an initial dose of $250 \mathrm{mg}$ / $\mathrm{kg}$ in male rats for antiurolithiatic impact. As a conventional comparison drug, cystone $(250 \mathrm{mg} / \mathrm{kg}$, p.o.) was used amount of different urolithiatic parameters in the biological samples i.e (urine, serum and kidney homogenate) and renal function were used as criteria for evaluating the antiurolithiatic impact of ELPA and ALPA after 28days of therapy span results indicated that, in a model similar to that of furosemide, the ELPA and ALPA (250mg $/ \mathrm{kg}$ ) elevate blood quantity and urinary excretion of electrolytes. ELPA and ALPA reduced the excretion and deposition of multiple urolithiatic parameters in ethylene glycol-induced urolithiatic model relative to urolithiatic command in a pattern similar to cystone control. Supplementation with ELPA and ALPA also avoids renal function deficiency. According to our studies, ELPA supports stronger anti-urolithic exercise.
\end{abstract}

Index Terms: Pisoniaalba, urolithiasis, ELPA, ALPA, cystone

\section{INTRODUCTION}

Since ancient times, humans obtained their ailments of remedies in nature. Especially, natural products naturally obtained and synthetically derived from them have found from varying plants worldwide, for prevention and treatment of different maladieshumanity[1]. Medicinal plants are used as agents of therapeutics since they have effective, cost effective changes in traditional medicine which are believed to be safe. But it actually have some side effects thus the belief is somewhat termed to be a misconception [2]. As an outcome, Based on concerns of public health, for proofing the safety of traditional medicinal plants before use. Experimental data on the toxicity of medicinal plants and extracts have to be collected for increasing safety for human uses and in pharmaceuticals growth [3]. Thus analysis of methodologies of medicinal plants for toxicity isthe common step for validating their use in applications therapeutically.

Revised Manuscript Received on July 22, 2019.

Kavitha Rani Mari, PunithavathiManogaran, AnandhiEswaran, Ph.D Research Scholars, Department of Biochemistry, Periyar University, Salem, India.

Dr.SuriyavathanaMthukrishnan, Associate Professor, Department of Biochemistry, Periyar University, Salem, India. Suriyabio14@ gmail.com
Urolithiasis is one of the disease that analyse in urology field which is not curable in allopathy. It is the kidney stone formation state (termed as nephrolithiasis) or and in any urinary tract parts, containing ureters (ureteral stone) and bladder (bladder stone). Urolithiasis has effect on the health care with prevalence of $>10$ percentage and expected rate of recurrence of 50 percentage approximately [4].The worldwide emergence of urolithiasis is more, and $80 \%$ or more of urinary calculi are stones of calcium oxalate $(\mathrm{CaOx})$ or $\mathrm{CaOx}$ with calcium phosphate [5]. Epidemiological analysis shows the nephrolithiasis is showing high prevalence in men $(12 \%)$ than in women $(6 \%)$ and is high in between the ages of 20 to 40 in both genders [6].

Various research, containing radiology and other lab methodologies are mostly not that useful to clear causes and stone forming mechanisms [7].Anyhow, recent investigations revealed that different factors can be the cause for stone formation. When the urine get saturated with insoluble particles due to more rate of excretion resulting in formation of crystals and stone formation by aggregation [8].Literature suggests, $P A$ leaves extract can be efficient in urolithiatic stage. Lack in scientific analysis reports its antiurolithiatic effect in induced experimental renal stone forming condition in lab animals. Thus currently the $P$. Alba extract analyzed against ethylene glycol initiated renal calculi in male rats (adult)is under taken.

\section{MATERIALS AND METHODS}

Chemicals

Ethylene glycol, Cystone, biochemical kits for serum and urine analysis were from Sigma Aldrich - Merck in India. All reagents and chemicals used are of analytical grade and chemical are from approved suppliers.

Plant material

The fresh Pisoniaalba leaves (Synonyms: Pisoniagrandis) are from Kolli hills located in Naamakal district, Tamil Nadu state, India. The plant collected, recognized by Prof. P Jayaraman, plant taxonomist, from plant anatomy research Centre (PARC), Chennai, Tamil Nadu. Certificate Reg No: PARC/2015/3251. The leaves collected were distilled water washed and the leaves were dried in shadow and fine powder I made by electrical blender.

Extraction of Pisoniaalba

The leaves of Pisoniaalba powder is made by using grinder to get coarse powder. Equal amount of powder can be sieved through 40 mesh sieve to get coarse powder of particle size desired. The powder was exposed to Soxhlet extraction with aqueous and ethanol $(95 \% \mathrm{v} / \mathrm{v})$ at $60^{\circ} \mathrm{C}$. Colorless solvent in the siphon tube was collected as the end of extraction.

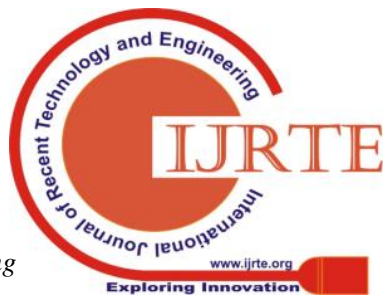


The extracts are concentrated by distillation to $3 / 4$ of its original volume. The extracts concentrated were taken in a china dish and evaporation done on thermostat controlled water bath till thick paste is formed. The extract is dried and stored in a glass bottle in a refrigerator at $4^{\circ} \mathrm{C}$. The dried, crude extracts concentrated were labeled as ALPA and ELPA. The yield was $12.8 \% \mathrm{w} / \mathrm{w}$.

Animals

Healthy male adult rats weighing 150 to $250 \mathrm{~g}$ of equal age groups were taken from Sree Venkateswara Enterprises, Bangalore, India, and the rats were housed randomly up on their arrival under hygienic conditions in cages of polypropylene to reduce stress of isolation, if any. The animals was of god ventilation and are at an optimum temperature of $25 \pm 4{ }^{\circ} \mathrm{C}$ containing 50 to $60 \%$ humidity relatively with $12 \mathrm{~h}$ dark and light cycle. The animals made adjusted to conditions in laboratory for one week prior to experiments and standard diet were given and also water ad libitum. The animals care and utilization in investigation process was allotted by the Institutional Animal Ethical Committee (IAEC), Rag No: (PU/IAEC/085/PO/OC/07/CPCSEA/BIOCHEM/07/2016),

Periyar University, Salem, Tamil Nadu, India, following guidelines of the 423 [OECD, 2001], Government of India. Acute toxicity studies

The acute oral toxicity investigation was done based on suggested technique by the OECD, 2001 guidelines for the acute toxicity assessment of plants with medicinal properties, with some alteration. Healthy adult in albino male rats weighing between 150 to $250 \mathrm{~g}$ were used. The animals were once at a time dosed and were randomly grouped into three within five. Grouplanimals received ad libitium only, while groups II and III were treated with ethanolic (ELPA) and aqueous extract (ALPA) of Pisoniaalba leaves at dose of $1000 \mathrm{mg} / \mathrm{kg}$ body wt. The animals were on standard diet $\&$ water .Extracts was orally given using gavages after overnight access to water. All animals analysis for common alteration in behavior were done ;toxicity symptoms, changes in mortality and body after therapy for $1 \mathrm{~h}, 24 \mathrm{~h}, 7^{\text {th }}$ day $\& 14^{\text {th }}$ day. After the observation periods all rats were sacrificed and serum collected for biochemical parameters assays (total albumin, bilirubin, protein, AST, ALT, ALP), and the internal organs were weighted.

\section{Ethylene glycol-initiated urolithiasis model in rats}

Ethylene glycol-induced urolithiasis samples were utilized to assess the urolithiatic activity in albino rats. Animal's separation into five various groups with six rats were done. The control (group 1) were on standard lab diet and water add libitum for 28days. Group II animals given with $0.75 \%$ of Ethylene glycol [EG] in water for 28 days using oral gauage. Group III with $0.75 \%$ of Ethylene glycol [EG] in water via oral transfer for 14 days; ELPA served for next 14 days. Group IV with $0.75 \%$ of Ethylene glycol [EG] in water via orally for 14 days; ALPA for next 14 days. Group V with $0.75 \%$ of Ethylene glycol [EG] in water via orally for 14 days; Cystone for next 14 days.

Collection and analysis of urine

After 28 days of an experiment, all animals were in individual metabolic cages with hydration $15 \mathrm{ml}$ urine and water samples were collected for 24 hours. Animalscan freely access to water throughout urine collection period. A drop of Hcl concentrated were added to urine collected and stored at $4^{\circ} \mathrm{C}$. After collection of urine, models usd for detrmining calciumcreatinine, , oxalate, protein,uric acid,urine volume, urinepH, and colour of urine.

Serum analysis

After collection of urine on $29^{\text {th }}$ day collectedblood was done under anestheticmild condition and cervical decapitation done for killing them. Serum divided by centrifugation at 12,000 rpm ,5 min and examined forphosphate, calcium, uric acid ,urea, and protein, creatinine, serum markar analysis (AST, ALT and ALP), serum enzymatic andnon enzymatic analysis by utilizing different biochemical kits (Span Diagnostics, Surat, India) by instruction of themanufacturer. Kidney homogenate Preparation and estimation in biochemical means

The abdomen was opened to collect 2 kidneys of each animal kidneys. Isolated were cleaned from extraneous tissue and ice-cold physiological saline and dried in hot air oven at $80^{\circ} \mathrm{C}$. A $100 \mathrm{mg}$ model of the kidney dried was boiled in $10 \mathrm{ml}$ of $1 \mathrm{~N}$ $\mathrm{Hcl}$ for $30 \mathrm{~min}$ and was homogenized. The centrifugation done at $2000 \mathrm{rpm}$ for $10 \mathrm{~min}$, and the supernatant was divided. The supernatant analysed for phosphate,calcium, urea, uric acid and creatinine, protein, albumin, bilirubin, renal tissue marker analysis, (AST, ALT and ALP), renal tissue enzymatic and non enzymatic anioxidant assays (CATcatalase; SOD - Super Oxide Dismutase; RG - Reduced Glutathione; GR - Glutathione Reductase; GST - Glutamyl S Transferase; GP - Glutathione Peroxidase) by utilizing different biochemical kits (Span Diagnostics, Surat, India) based on instruction of manufacturer.

Histopathology examination

After 28 days the rats were administered with anesthesia to avoid pain and all groups of rats were scarified and abdomen was open to collect the kidney. Kidneys carefully removed and phosphate buffered saline ( $\mathrm{pH}$ 7.4) wash were done. For pathological study the kidneys were fixed in $10 \%$ formaldehyde solution. The tissues fixed were embedded and processed in paraffin. Thin sections cut at a thickeness of $5 \mu \mathrm{m}$ by microtome and placed in slides. The slides staining were with Haemotoxylin and Eosin for histopathological analyses (H\&E). The sections were then examined under microscope at different magnification. Calcium deposition in intratubular renal tissue and other parts was performed.

Statistical analysis

The results obtained from the invivo animal model were expressed as mean \pm SD. The significant changes between control and EG inducer groups and treated groups were assessed by one way ANOVO method followed by DMRT (Deccan's Multiple Range Test) by statistical Package of Social Science (SPSS) software version package 16 for windows. Values would be significant statistically when $\mathrm{P}$ less than 0.05 .

\section{RESULTS}

Acute toxicity test

The acute toxicity study is crucial for evaluating effects of extracts or compounds, after 1 oral intake [Khan et al., 1982]. In the acute toxicity studies, the Pisoniaalba leaves extracts (ELPA and ALPA) administered orally (1000 mg/kg) don't have any toxicity signs or mortality in male rats throughout period of observation of fourteen days. 
Also, the animals given with extract don't show significant changes in body weight in period of experimental.table-1, shows extract have no effect in normal animal's growth. As mentioned, oral administration of Pisoniaalba leaves extract $1000 \mathrm{mg} / \mathrm{kg}$ noted to safe whereby did not cause any signs of mortality.

Table 1.Acute toxicity study of ELPA and ALPA

\begin{tabular}{|l|c|c|c|c|}
\hline Parameter & $\mathbf{1} \mathbf{H}$ & $\mathbf{2 4} \mathbf{H}$ & $\mathbf{7}^{\text {th }}$ Day & $\mathbf{1 4 ^ { \text { th Day } }}$ \\
\hline Appearance & N & N & N & N \\
\hline Activity & P & P & P & P \\
\hline Gait & N & N & N & N \\
\hline Reaction to Stimulus & & & & \\
a) Sound & + & + & + & + \\
b) Touch & + & + & + & + \\
c) Light & + & + & + & + \\
\hline Lacrimation & A & A & A & A \\
\hline Salivation & A & A & A & A \\
\hline Piloerrection & A & A & A & A \\
\hline Stimulation & A & A & A & A \\
\hline Depression & A & A & A & A \\
\hline Convulsions & A & A & A & A \\
\hline
\end{tabular}

A- Absent; N- Normal; P- Present; ++ - Present medium + minimum;

Modern therapeutic and diagnostic aids like extrarectoporeal shock, utroscopy, nephrolithiotomy had rellalunized the urological practices but can't alter the repeated formation of stone and have its side effects. urolithiasis is multi factorial disease that involve in biochemical pathways alterations, so currently demand for new therapy mode is increasing .latest studies enlightened effectiveness of traditional plants and natural product for treating and managing urolithiasis. However remedies of herbal means to possess multiple bioactive components through different pathways like antialgeric, diuretic antioxidants, etc. Pisoniaalba leaves have been known for its medicinal property in china India, and European countries.

The leaves have been reported to possess this multiple pathways. Pisoniaalba leaves extracts aqueous and ethanolic are used in renal ailments treatment in some areas of India. But antiurolithiatic effect of this is not scientifically proven so far. Thus this study is aimed to ascertain ethnic practice in a scientific mode of utilizing the Pisonia alba leaves extracts in order to rationale its toxicity level and to ascertain the maximum acceptable limit as a drug in the process of treatment in ethylene glycol induced urolithiatic rat model. This acute toxicity study clearly exhibited the margin of safety in order to use this source for oral administration to the animals.

The table 3 depicts the biochemical parameters analyzed in the animals treated with ELPA, ALPA leaves extracts. The assessment of albumin, bilirubin and protein levels exhibit that the content bilirubin is noted to be normal followed by albumin and protein. The treated animals groups did not signified a drastic change in the biochemical parameters in comparison to control group (I) is explained by the statistical analysis.

Albumin is the most significant plasma protein its level in serum is a key significant prognostic factor in radical kidney disease and it also displace free radicals scavenging process. Bilirubin is a catabolite of heme. It formed from the lysis of red cells within the reticulo endothial frame work which is the conjugated protein with albumin. The treated group ELPA and ALPA did not showed significant alteration in the biochemical components when compared with control group.

From the statically analysis the results arrived exhibits that the serum markers AST, ALT, ALP of the groups treated in comparison to control group, no marked difference in levels with the control is seen which signifies that Pisoniaalba leaves extracts seems to be maintain the normal effect where by outlays its margin of safety and non toxic. Transaminases are important hepatic and renal damage markers, it and increase and elevated in the blood stream, it is a strong indicator for renal and hepatic injury. Additionally, the results Table 3 showed that the ELPA and ALPA leaves extracts maintained normal renal function in rats.

Data presented in table 4 represents the organs namely liver, heat, kidney and their respective weight when compared with control the treated groups ELPA and ALPA did not show any significant difference in their organ weight. From the table4 value inference drawn on the organ weight especially kidney by the treated groups ELPA and ALPA noted to be safe and non toxic.

Table 2.Biochemical analysis in toxicity study of ELPA and ALPA

\begin{tabular}{|c|c|c|c|}
\hline Categories & Control & ELPA & ALPA \\
\hline \multicolumn{4}{|c|}{ Biochemical analysis } \\
\hline Albumin (g/L) & $3.93 \pm 0.14$ & $3.85 \pm 0.10^{\circ} \mathrm{ns}$ & $3.77 \pm 0.08^{=n 5}$ \\
\hline Bilinubin(g/L) & $0.33 \pm 0.20$ & $0.56 \pm 0.13^{\circ} \mathrm{ns}$ & $0.51 \pm 0.05^{=\mathrm{n}}$ \\
\hline Protein $(g / L)$ & $6.56 \pm 0.1$ & $6.78 \pm 0.09^{*} \mathrm{~ns}$ & $6.45 \pm 0.1^{=n 5}$ \\
\hline \multicolumn{4}{|c|}{ Serum markers } \\
\hline $\mathrm{AST}(\mathrm{U} / \mathrm{L})$ & $45.16 \pm 1.91$ & $49.78 \pm 2.15^{*} \mathrm{~ns}$ & $47.29 \pm 1.42 \# \mathrm{~ns}$ \\
\hline $\mathrm{ALT}(\mathrm{U} / \mathrm{L})$ & $53.07 \pm 1.77$ & $56.35 \pm 1.83^{*} \mathrm{~ns}$ & $54.54 \pm 1.22=\mathrm{ns}$ \\
\hline $\mathrm{ALP}(\mathrm{U} / \mathrm{L})$ & $112.84 \pm 5.73$ & $122.24 \pm 4.03 * \mathrm{~ns}$ & $115.14 \pm 4.76=\mathrm{ns}$ \\
\hline
\end{tabular}

All values shown as mean $\pm \operatorname{SEM}(n=6)$; *ns - no any significant changes control vs. ELPA groups, \# ns - no any significant changes control vs. ALPA groups

Table 3. Weights of Organ of rats in study of toxicity of ELPA and ALPA

\begin{tabular}{|c|c|c|c|}
\hline Organs & Control & $\begin{array}{l}\text { ELPA } \\
(1000 \mathrm{mg} k \mathrm{k} . \mathrm{wgt})\end{array}$ & $\begin{array}{l}\text { ALPA } \\
(1000 \mathrm{mg} \mathrm{kb} . \mathrm{wgt})\end{array}$ \\
\hline Heart(g) & $0.86 \pm 0.06$ & $0.93 \pm 0.04^{\circ} \mathrm{nt}$ & $0.81 \pm 0.06^{=15}$ \\
\hline Liver (g) & $7.70 \pm 0.17$ & $7.77 \pm 0.23^{\circ} \mathrm{ns}$ & $7.50 \pm 0.25^{ \pm 15}$ \\
\hline $\operatorname{Renal}(\mathrm{g})$ & $1.87 \pm 0.08$ & $1.92=0.08^{*} \mathrm{~ns}$ & $2.01=0.09^{=15}$ \\
\hline
\end{tabular}

Wet weight of the organs were reported as mean \pm SEM $(\mathrm{n}=6)$; *ns, significant changes is not seen in in control vs. ELPA group, \# ns, significantly no change seen between control and ALPA group

\section{Effect of ELPA and ALPA on urolithiasis-induced by ethylene glycol}

Effect of ELPA and ALPA on volume of urine weight of body, and weight of kidney

Ethylene glycol administration (group II $-0.75 \% \mathrm{v} / \mathrm{v}$ water) reduce body weight of control urolithiatic rats (group II). That changes are prevented in ELPA cystone, and ALP treated group (III, IV, and V) in comparison to (group II).

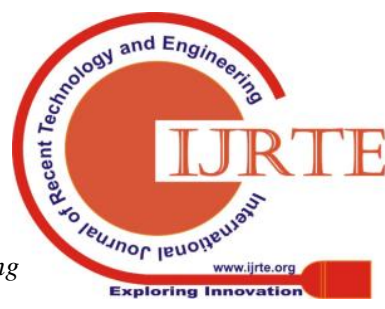


Reduction in 24hours volume of urine $(\mathrm{ml})$ was significantly noted in urolithiatic control of rats and in case of ELPA and ALPA improves in urinary output in comparision to urolithiatic control animals table 6. Furthermore, increase was there in kidney's wet weight (g) in urolithiatic control rats, which was importantly prevented by ELPA, ALPA and cystone. The physical parameters namely the body wt, urine $\mathrm{pH}$, urine volume were drastic alteration in the level of urine and $\mathrm{pH}$ which is acidic and reduced volume in the Ethylene glycol groups (II). On treatment with Pisoniaalba leaves extract for experimental periods the animals restored the body. wt followed by increase output of urine volume and stabilization of acidic to basic nature of urine were determined by this physical assessment.

Table 4.Effect of Pisoniaalba leaves on different physical parameters urolithiasis induced by ethylene glycol in rats.

\begin{tabular}{|c|c|c|c|c|c|c|c|}
\hline & \multicolumn{3}{|c|}{ UrinepH } & \multicolumn{3}{|c|}{ Urine rolume } & \multirow{2}{*}{\begin{tabular}{|l} 
Bodyut \\
Bodynt \\
(ghastt) \\
day28
\end{tabular}} \\
\hline & DayO & Day $14^{\text {th }}$ & Day 28 & $\begin{array}{l}\text { Day0 } \\
(\mathrm{m} / 24 \mathrm{~h})\end{array}$ & $\begin{array}{l}\text { Day } 14^{\text {th }} \\
(\mathrm{m} / 2 \mathrm{hh})\end{array}$ & \begin{tabular}{|l|} 
Day 28 \\
$(\mathrm{~m} / 24 \mathrm{~h})$
\end{tabular} & \\
\hline Control & $7.0 \pm 0.58$ & $7.0 \pm 0.54$ & $7.3=0.79$ & $5.5=0.7$ & $7.5 \pm 0.64$ & $6.8=0.78$ & $262 \pm 1.3$ \\
\hline$E G$ & $7.2 \pm 0.67$ & $5.5 \pm 0.59^{\circ}$ & $4.8 \pm 0.499^{\circ}$ & $5.7 \pm 0.66$ & $8.2 \pm 0.68^{\circ}$ & $8.8=0.67 \mathrm{~h}^{\mathrm{h}}$ & $200=1.7^{\prime}$ \\
\hline EIPA & $7.1 \pm 0.66$ & $5.2 \pm 0.78^{\circ}$ & $6.6=0.48^{\circ}$ & $5.0 \pm 0.83$ & $7.2 \pm 0.52^{\circ}$ & $6.8=0.587$ & $228 \pm 1.1^{7}$ \\
\hline ALPA & $7,3 \pm 0.7$ & $4.8 \pm 0.87^{\circ}$ & $6.8 \pm 0.0 .56^{\circ}$ & $4.9=0.43$ & $5.5 \pm 0.49^{\circ}$ & $5.0 \pm 0.622^{2}$ & $262 \pm 1.3^{\circ}$ \\
\hline Cystone & $7.1 \pm 0.76$ & $5.0 \pm 0.79^{\circ}$ & $6.6 \pm 0.55 f^{t}$ & $5.6=0.56$ & $8.54=0.5^{\circ}$ & $7.6=0.522^{\circ}$ & $241 \pm 1.5^{5}$ \\
\hline
\end{tabular}

All the values are reported as mean $\pm \operatorname{SEM}(n=6)$. *p less than 0.05 , vs day $14 ; \uparrow \mathrm{p}$ less than 0.05 , vs day 28 ; final weight of body of the control, EG $(*)$ vs treated groups were (\#, $f, \$)$.

Effect of ELPA and ALPA on urine analysis

The biochemical analysis of ELPA and ALPA group's data (table 5) provides effective note that the calcium, uric acid, phosphate, levels in the groups induced by ethylene glycol showed enhanced level on treatment with ELPA and ALPA extracts. The enhanced level of this parameter were significantly reduced and restored to normalcy when compared to the control. Parelly the content of keratinize, protein and oxalate also exhibited the similar result analysis where by the effectiveness of Pisoniaalba leaves extracts in both aqueous and ethanolic leaves extract reveals and it upholds the potential therapeutic bioactive compounds indulged in the drastic alteration and recovering to the normal level. Also, results can be compared with, cystone the standard drug that which also reduced urolithiatic promoters significantly in y excretion of urine of urolithiatic control rats Effect of ELPA and ALPA on serum analysis

The activity of ELPA and ALPA on serum biochemical promoters were expressed in table 5. The probability changes between the control (I) vs. EG group (II) and treated groups (ELPA-III, ALPA-IV, and cystone-V) were clearly depicted in the table. In current study, the ethylene glycolalone treated rats showed highest elevation of various biochemical analysis like oxalate, phosphate, calcium, uric acid, protein and creatinine in comparison with normal rats which are the control. Anyhow, ELPA and ALPA with ethylene glycol shows reduction in oxalate, serum calcium, phosphate, protein, creatinine and uric acid, in comparision with controls which are ethylene glycol alone-treated and the results are compared to observed value of standard drug, cystone (Table 5).Serum enzymatic antioxidants analysis is statically defined and depicted the table 5. This table demonstrates that ELPA and ALPA treated groups showed positive distortion in the enzymatic antioxidant levels when compare to the urolithiasis induced by ethylene glycol in rats. effectiveness of treated groups with both aqueous and ethanolic leaves extract of Pisonia alba signifies that this particular source possess good store of protective antioxidant enzymes (catalase, super oxide dismutase, reduced glutathione; glutathione reductase; glutamyl S transferase; glutathioneperoxidase) which may be the prime factor to promote therapeutic effect against urolithiasis. The oxidative stress induced by the ethylene glycol chemical toxicant in the renal system whereby leads to leakage of the these antioxidants countering the free radical scavenging mechanism which has been positively expressed by the enhanced level of the antioxidant enzymes by the influence of the Pisonia alba leaves extract, which possess valuable phytoconstituents having the restoration effectiveness of antioxidant enzymes in experimental animal group which is clearly exhibited by this study. In this analysis protein level shows no detrimined alteration in ELPA and ALPA treated rats Group in relation to the Control rats Group. It represents the non-toxic effect of ELPA and ALPA on protein

Effect of ELPA and ALPA on analysis of kidney homogenate

The deposition of the urolithiatic parameters in the renal tissues specifically Calcium, Oxalate, Phosphate, protein, creatinine, uric acid were registered and represented in the table7. The biochemical parameters of the renal tissue shows that the ethylene glycol induced group II exhibited drastic increase in their levels on for the treatment with Pisoniaalba leaves extract both aqueous and ethanolic showed a remarkable reduction and restoration to the normal range on comparing with the control group. This positive mode of alteration I the biochemical constituents and restoring into normal emphasize the Pisoniaalba leaves possess effective bioactive compounds which may be responsible to alter the mechanism restoring the biochemical constituents in the real tissues.

The condition of urolithiatic is dependent on the calcium oxalate stone formation in urine super saturation result in calcium salts formation. Some metabolic abnormalities are also noted like hyperoxalouria, hypercalciuria, and hypocitraturia could vary Protein composition of urine which enhance prosperity of stone formation. In the current study ethylene glycol administered rat significantly increase excretion calcium oxalate and uric acid in 24hrs urine sample after the oral induction of ELPA and ALPA. The urinary biochemistry remarkably improved and results in significant low level uric acid and calcium oxalate in renal content and fewer renal stones were also noted which results in the effectiveness of Pisonia alba leaves serving as a antiurolthiaitc component $t$ in dissolution of renal stone which is further supported by our histopathological experimental study.

The datas analyzed statically presented in the table 5 which represents the renal tissue marker enzyme levels (AST, ALT, ALP) of ELPA and ALPA treated groups against ethylene glycol induced groups II. The marked reduction in the level of tissue markers ma due to the oxidative damage induced by the ethylene glycol leads to renal tissue damage 
whereby the membrane content or leaked out to the ECF (extra cellular surface) which is noted from the table 7. On the treatment with ELPA and ALPA extracts potentially regenerated the cell growth where by the tissue regeneration marked with the enhanced level of marker enzyme in the rerated groups which signifies Pistoia alba leaves has vital and protective resorting effect in membrane regeneration and restoring renal tissue damage which is the positive indication of antiurolithiatic effect of Pisonia alba leaves.

The varied alteration in the physical and biochemical and marker enzyme assay determined that the induction and formation of urinary stone by using ethylene glycol causes very drastic oxidative cellular damage leads to chronic renal especially glomerular and tubular renal tissue damage which has been very clearly studied in the histopathological experimentation . the damage imitated by the free radicals has been counter acted very effectively on treatment with the ELPA and ALPA extracts which emphasize the potential store of vital antioxidant enzymes which has rich effectively clears the free radicals which has been produced during the ethylene glycol induction table 5. The altered metabolic and biochemical pathways in the animal has been clearly imagined with the specific parameters determined and analyzed to exhibit the effective antiurolithiatic property of Pisonia alba leaves which is indulged has been exploited this systematic analysis the antioxidant status which is an important therapeutic tool to later server as an effective and natural pharmacological and bioactive compounds with margin of safety which has been outlayed in Pisonia alba leaves as an antiurolithiatic natural source.

Table 5.Effect of ELPA and ALPA on urine, serum and renal homogenate parameters in Ethylene Glycol induced Albino rats.

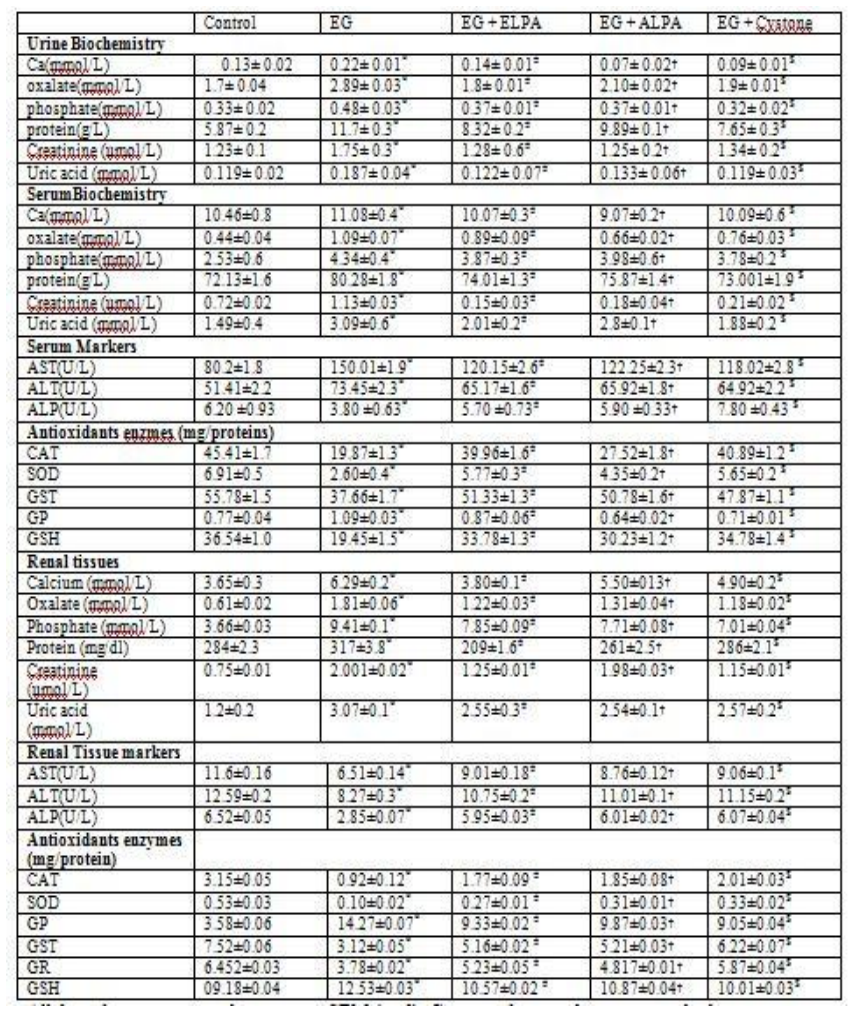

All the values are mean \pm SEM $(n=6)$. Cat - catalase; sod super oxide dismutase; RG - reduced glutathione; GR glutathione reductase; Gst - glutamyl s transferase; Gp glutathione peroxidase,All the values are reported as mean \pm $\operatorname{SEM}(\mathrm{n}=6)$. * p less than 0.05 , vs control group; \# $\mathrm{p}$ less than
0.05, vs EG groups; † p less than 0.05, vs. EG groups; \$ p less than 0.05 , vs. EG groups dismutase (SOD): Inhibition of $50 \%$ of nitrite $/ \mathrm{min} / \mathrm{mg}$ protein ;Catalase (CAT): $\mu$ moles of $\mathrm{H}_{2} \mathrm{O}_{2}$ decomposed $/ \mathrm{min} / \mathrm{mg}$ tissue ; Glutathione peroxidase (GPx): $\mu \mathrm{g}$ of glutathione consumed $/ \mathrm{min} / \mathrm{mg}$ protein; Glutathione-S-transferase (GST): $\mu$ moles of CDNB-GSH conjugate formed/min/mg protein ; Glutathione reductase (GR): $\mu$ moles of NADPH oxidised/min/mg protein; GSH: $\mu \mathrm{g} / \mathrm{mg}$ of proteins

Histopathology examination

Microscopic examination of the renal tissue stained with haematoxylin and eosin of experimental rats depicts that pathological group of control had incremented deposition of calcium crystals in comparision to the normal\& standard drug control group, with no crystal deposits. Pisoniaalba treated groups ( $3 \& 4$ ) $250 \mathrm{mg} / \mathrm{kg}$ b.wt showed minimum crystal presence than with EG group. ELPA and cystone group (3\& 4) was found to increase the minimum deposition of formation of crystals with the crystal deposits clearance from renal tubules on pair with the standard drug control groups as shown in Fig 1.Histopathologicalmanifestation additionally bolster the above outcomes. Organization of Pisonaalba leaves extract to ethylene glycol rodents averted super immersion of calcium oxalate and in this way diminished their testimony in renal tubules.
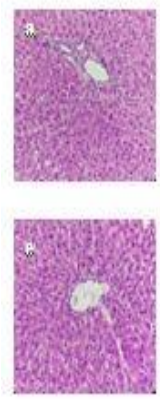
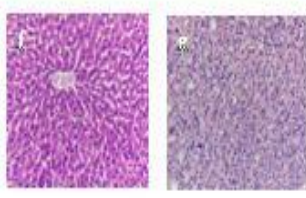

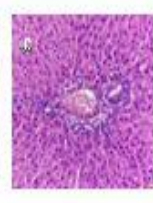

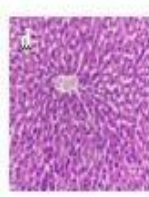

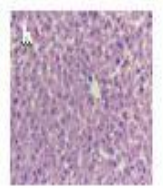

Fig-1Crystal examination in renal tissue under microscope: (a): group (1) control with normal conditions; (b): EG group for 14 days, crystallization with inflammation; (c): EG group (2) served for 28 days, crystallization with severe inflammation; group (3) received ELPA $250 \mathrm{mg} / \mathrm{kg}$ body wgt express less inflammation with lowest crystal deposition; group (4) received ALPA $250 \mathrm{mg} / \mathrm{kg}$ body wgt express the less inflammation with lowest crystal deposition.

\section{DISCUSSION}

In the current investigation, we described antiurolithiatic activities of extract of Pisoniaalba leavesin experimentally induced animal samples. At first, the acute toxicity of ELPAand ALPA (1000 mg/kg) is taken to introduce chronic antiurolithiatic activity's effective dose. Analyzed Weight loss in urolithiatic rats is owing to disturbances in carbohydrates, metabolism of proteins or fat and anorexia that is dependent on ethylene glycol ingestion. The current investigation displayed important decrease in body weight of urolithiatic rats that was efficiently suppressed by ELPA and ALPA therapy. Additionally, urine volume was also reduced in urolithiatic rats that owing to stone and crystal formation in renal tissue. 
Reports shows urinary excretion volume increase improves the elimination of small crystals and decreases the tendency of crystals to aggregate or grow. Anyhow, Eland ALPA supplementation enhances the excretion of urine volume and thus hastens the removing of stones which are pre-formed and prevent formation of new stone in urinary system. Hype in wet and dry weight of kidney also noted in in urolithiatic control rats owing to various urolithiatic promoters deposition in kidney, Also ELPA and ALPA decrease weight of kidney by suppressing the various urolithiatic promoters deposition in kidney. The current investigation also displays that there is a oxalate and calcium levels increase in kidney homogenate by treatment of ethylene glycol in urolithiatic rats. Otherwise, ethylene glycol increase the oxalate and calcium deposition in renal tissue that was attenuated by administration of ELPA and ALPA. The calcium level increase in renal tissue might be owing to bioavailability of nitric oxide (NO) increase which activates cGMP (30, 50-cyclic guanosine monophosphate) that which controls the intracellular calcium level increase.

\section{CONCLUSION}

Acute toxicity studies were done to ensure safety of Pisoniaalba leaves in male rats. General behavior and signs were seen to be normal in animal tested till $1000 \mathrm{mg} / \mathrm{kg}$, in repeated dose of 28 days followed by investigation for acute toxicity, no variation in biochemistry and blood parameter values where found for extract treated \& control animals. Based on this, the Pisoniaalba leaves ethanol extract was considered safe till $1000 \mathrm{mg} / \mathrm{kg}$ in male rat. ELPA and ALP shows protection from urolithiasis induced by ethylene glycol suppressing strongly the urolithiatic promoters in kidney tissue, urine, and serum. In addition, thus, it emphasize that the leaves of Pisoniaalba possess medicinal property and property to prevent renal stone. Detailed investigation is to be done for explaining Pisoniaalba leaves's mechanism(s) of action.

\section{ACKNOWLEDGEMENTS}

This part of the work got support from grants from Rajiv Gandhi National Fellowship (RGNF-SRF) by UGC and the authors are thankful to Periyar University.

\section{REFERENCES}

1. Banakar et al., 2004 M.C. Banakar, S.K. Paramasivan, M.B. Chattopadhyay, S. Datta, P. Chakraborty, M. Chatterjee, K. Kannan, E. Thyagarajan, 25-dihydroxyvitamin D3 prevents DNA damage and restores antioxidant enzymes in rat hepatocarcinogenesis induced by diethylnitrosamine and promoted by phenobarbitolWorld $\mathrm{J}$. Gastroenterol. 10 (9) (2004), pp.1268-1275.

2. Bello , I. Bello, A.S. Bakkouri, Y.M. Tabana, B. Al-Hindi, M.A Al-Mansoub, R. Mahmud, M.Z. Asmawi, Acute and sub-acute toxicity evaluation of the methanolic extract of Alstoniascholaris stem bark, Med. Sci.

3. Bhatt and Paul, 2008 P. Bhatt, P. Paul, Analysis of urinary stone constituents using powder X-raydiffraction and FT-IR, J .Chem. Sci. 120 (2) (2008), pp.267-273.

4. Butterweck, and Khan, 2009 V. Butterweck, S.R. Khan, Herbal medicines in the management of urolithiasis: alternative or complementary, Planta.Med, 75 (2009), pp.1095-1103.

5. Calixto et al., 1998 J.B. Calixto, A.R. Santos, V. CechinelFilho, R.A. Yunes, A review of the plants of the genus Phyllanthus: their chemistry, pharmacology, and therapeutic potential, Med. Res. Rev. 18 (1998), pp.225-258
6. Dhanasekar and Sorimuthu, 2005 S. Dhanasekar, S. Sorimuthu, Beneficial effects of Momordicacharantia seeds in the treatment of STZ-induced diabetes in experimental rats, Biol. Pharm. Bull.28 (2005), pp.978-83.

7. Divakar et al., 2010 K. Divakar, A.T. Pawar, S.B. Chandrasekhar, S.B. Dighe, G. Divakar, Protective effect of the hydro-alcoholic extract of Rubiacordifolia roots against ethylene glycol induced urolithiasis in rats, Food.Chem.Toxicol, 48 (2010), pp.1013-1018.

8. Evan, 201 A.P. Evan, Physiopathology and etiology of stone formation in the kidney and the urinary tract, Pediatr.Nephrol. 25 (2010), pp. 831-841. HT, 1996 H.T. 